\title{
Las desventajas de la Política \\ Comparada en América Latina: \\ énfasis en el caso Argentino
}

The disadvantages of Comparative

Politics in Latin America: emphasis

on the Argentinian case

\section{Martín D'Alessandro}

\section{Resumen}

La ciencia política de América Latina viene experimentado un debate mal orientado acerca de sus enfoques teóricos y estrategias metodológicas. El artículo recoge parte de ese debate para argumentar que la política comparada regional se ve desfavorecida debido a una serie de creencias erróneas acerca de su relevancia, método y presencia en el campo disciplinar. Se abordan empíricamente las dimensiones más importantes del desarrollo de la política comparada en la región (con énfasis en el caso Argentino) para rebatir los argumentos que le adjudican predominio y hegemonía en el campo politológico regional y local.

\section{Palabras clave}

Ciencia Política; Política Comparada; América Latina; Argentina.

\begin{abstract}
Latin American political science has experienced a wrong-driven debate on its theoretical approaches and its methodological strategies. This article aims to participate of that debate arguing that regional comparative politics is negatively affected by it due to some wrong assumptions on about relevance, methods and presence in the political science field. It empirically addresses the most important dimensions of comparative politics in the region (with emphasis on the Argentinian case) to refute the argument that suggest its predominance and hegemonic position in Latin American political science field.
\end{abstract}

Keywords

Political Science; Comparative Politics; Latin America; Argentina. 


\section{Introducción}

El desarrollo de la ciencia política en América Latina es dispar y heterogéneo. Por esa razón, la relevancia (o incluso la presencia) de la política comparada es tan disímil en los diferentes países de la región que hace una empresa riesgosa tratar de identificar cuáles son los problemas, temas y prioridades de la agenda de la política comparada regional en lo que atañe a la investigación y la docencia. Sin embargo, algunas breves reflexiones, necesariamente generales, pueden contribuir al debate sobre el tema. En la primera sección de este artículo se argumenta que la región ha absorbido livianamente el debate iniciado por Sartori sobre hacia dónde va la ciencia política. En la segunda sección se refutan algunas ideas comunes acerca de la relevancia de la región en la política comparada mundial. En la tercera, se abordan empíricamente las dimensiones más importantes del desarrollo de la política comparada en la región (con énfasis en el caso argentino) para rebatir los argumentos que le adjudican predominio y hegemonía en el campo politológico regional y local. El cuarto y último apartado aborda, más optimista, el tema de la incidencia pública de la política comparada.

\section{El debate en el centro de la ciencia política}

En los ámbitos en los que se desarrolla la ciencia política latinoamericana es bastante usual encontrarse con posiciones diversas respecto de la manera en que se produce y transmite el conocimiento de la disciplina. En términos generales, las posiciones centrifugan hacia dos posiciones típico-ideales. De un lado se ubica la ciencia política empírica, teóricamente inclinada hacia enfoques racionalistas y/o neoinstitucionalistas, y metodológicamente inclinada hacia la cuantificación y/o la comparación; y en el otro extremo se sitúa la ciencia política lindera con la filosofía política, de corte más normativo y/o metodológicamente orientada hacia el estudio profundo de casos o fenómenos particulares con datos cualitativos más o menos sistematizados. Unos ponen énfasis en el carácter científico de la disciplina mientras que los otros lo hacen en su carácter político. Sin embargo, como se intentará argumentar en este trabajo, este es un debate mal orientado.

Recientemente, una de las causales de esta mala orientación fue la lectura superficial del texto que uno de los padres de la disciplina, Giovanni Sartori, publicó en 2004 (SARTORI, 2004) ${ }^{1}$. Allí Sartori sigue manteniendo sus posiciones históricas (ver, por ejemplo, Sartori, 1984) a favor de una ciencia política como disciplina autónoma, con un lenguaje especializado, con

\footnotetext{
${ }^{1}$ El texto, junto con algunas reacciones a las que también haremos referencia, fue publicado originalmente en PS Political Science and Politics (vol. 37, n. 4, 2004), la revista de carácter más institucional que académico de la American Political Science Association. El debate completo fue también publicado en castellano en la revista mexicana Política y gobierno. El texto de Sartori también puede encontrarse en la Revista Española de Ciencia Política (n. 12, abril, 2005).
} 
definiciones, con precisión metodológica, separada de la narrativa, del derecho y la filosofía, pero agrega que no está a gusto con "el molde" de la ciencia política estadounidense actual. Según él, lo que se volvió demasiado estrecho es la noción de ciencia en Estados Unidos, con sofisticadas técnicas de investigación y con desarrollos estadísticos complejos, que muchas veces da poco valor a la lógica y a la definición de una metodología disciplinar propia. En resumen, Sartori no está en contra de los métodos cuantitativos, sino de contar sin pensar, y de no usar la lógica al pensar. Y critica, por otro lado, que el saber de la ciencia política se concentre en el plano de la producción de un conocimiento que no tiene como horizonte o meta final el ser aplicado en la práctica, y por lo tanto, verificado o falseado en su aplicación.

En el debate originalmente publicado, hay dos respuestas en desacuerdo a Sartori. David Laitin (profesor en la Universidad de Stanford y ex vicepresidente de la American Political Science Association) le replicó que la ciencia política actual tiene mucha vitalidad, y que no se descuidan las diferentes dimensiones de la disciplina. Así, los programas de investigación abordan tanto el campo normativo (por ejemplo, cómo debería definirse la igualdad) como el campo analítico (por ejemplo, qué sistemas electorales ayudan a producir legislación de una manera más democrática ${ }^{2}$ ) y el campo empírico (por ejemplo, en comparaciones previstas por autores como Karl Deutsch, Seymour Lipset y Stein Rokkan, tan clásicos como el propio Sartori). De esta forma, argumenta Laitin (2004), se obtuvieron certezas muy relevantes tanto para la disciplina como para la vida política. Por ejemplo, Adam Przeworski mostró que el desarrollo económico no hace nacer a la democracia, pero hace más probable que sobreviva; Charles Boix mostró que las democracias no nacen al azar sino que surgieron en condiciones de relativa igualdad económica; y otros autores mostraron que el orden político (es decir, la ausencia de guerra civil y/o de colapso estatal) aumenta cuando hay presencia de factores como el bienestar económico y la capacidad para controlar la insurgencia, con muy poca relevancia de las variables culturales. El problema que confunde a Sartori, sigue Laitin, es que muchas veces las contribuciones sobre resultados políticos relevantes (como justicia, representación, orden, democracia) se pasan por alto porque la disciplina no tiene un núcleo teórico aceptado (como lo tienen la física o la economía) ni un método consensuado (como lo tienen la biología o la psicología).

La otra respuesta fue la de Josep Colomer (2004). Para este autor, la poca investigación aplicada por la que se queja Sartori se debe a que la ciencia política como disciplina académica se encuentra en un estado bastante atrasado: es normal que en el desarrollo de las ciencias haya una primera etapa de clarificación de las definiciones (a lo que Sartori, como uno de los fundadores de la disciplina, se ha

\footnotetext{
2 "Si bien esos artículos emplean modelos crípticos y pruebas estadísticas complejas, son parte de un programa de investigación común que intenta responder preguntas fundamentales en la teoría democrática" (LAITIN, 2004, p. 365).
} 
dedicado con enorme influencia), luego una de mediciones cuantitativas, luego una de hipótesis causales, y finalmente una etapa, que todavía no ha sobrevenido, de teorías explicativas. Según Colomer (2004), a pesar de algunos fundamentos teóricos importantes aportados por autores como Duverger, Dahl, Downs, Olson y Riker, un ejemplo claro de que la investigación teórica tiene cuestiones muy básicas aún no resueltas es el papel fundamental que siguen teniendo (y la inusual devoción que siguen inspirando) los autores clásicos de la historia de la ideas, muchos de cuyos conceptos y argumentos son confusos y ambiguos.

Este debate, deudor de una vieja controversia sobre el estatus de las ciencias sociales en general (ROCHA, 2012), es apasionante para los politólogos, pero en América Latina es apenas incipiente, puesto que es necesario previamente encarar una discusión seria acerca de la identidad de la disciplina dentro de las ciencias sociales y de las metodologías que utiliza para proveer el avance del conocimiento científico (NEGRETTO, 2004). Un ejemplo paradigmático de la interpretación liviana y efectista de este debate es el libro del politólogo mexicano César Cansino (2008). Cansino dice que el trasfondo que anima su libro es la crítica de Sartori, pero paradójicamente, para sostener algo bien distinto a lo que sostiene Sartori: Cansino identifica lo empírico solamente con lo formalizado o matematizado, y cuestiona la autonomía de la ciencia política respecto de la filosofía política. No aclara que Sartori discrepa con "el molde" de la ciencia política estadounidense actual pero no con la ciencia política como disciplina autónoma, dotada de precisión metodológica y separada de la filosofía, tres características que Cansino da por perimidas, casualmente diciendo que lo hace "en sintonía con el tono crítico de Sartori” (2008, p. 8-9).

A pesar de reconocer algunas de las ventajas metodológicas de la política comparada, Cansino (2008) le achaca a esta subdisciplina su carácter institucionalista actual, y en particular, critica a los estudios sobre la calidad de la democracia por pretender una objetividad al estilo positivista-conductista y el no asumir la tarea de ofrecer criterios sugerentes para lograr que las sociedades del futuro puedan ser más democráticas que en el presente. Según este autor, entonces, la ciencia política empírica debería no pretenderse la única "científica" y reconocer al fin los contenidos valorativos inmersos en sus propias definiciones. Finalmente, dedica varios capítulos a acusar a los estudios sobre la calidad de la democracia de no dar cobertura teórica al "resurgimiento de la sociedad civil": movimientos, reclamos y luchas ciudadanas que dan contenido a la política real.

Mi impresión es que Cansino (2008) ha llevado el (mal orientado) debate original a un punto extremo, en el que termina por adjudicar a la ciencia política empírica el cometer todos los pecados posibles. De hecho, y sin ir más lejos, una lectura un poco más atenta de Guillermo O'Donnell, uno de los fundadores y referentes de los estudios sobre la calidad de democracia, desmentiría esas afirmaciones de manera inmediata. En su último libro, que condensa los trabajos de sus últimos veinte años, O’Donnell (2010) se centra en las interrelaciones entre 
el Estado, el régimen y los individuos. Esta articulación trasciende los análisis que Cansino critica, y ha sido abordado por O'Donnell en varios artículos anteriores también importantes (D'ALESSANDRO, 2011). Los ejes de todos estos trabajos convergen en la situación de pobreza de vastos sectores de la población latinoamericana, la imposibilidad del Estado de hacer regir la ley en todo su territorio, su articulación con los rincones menos democráticos del régimen y la concomitante limitación a los seres humanos en su dignidad de ciudadanos. La vinculación del Estado - y sus manifestaciones físicas, como el territorio o la burocracia, y simbólicas, como la identidad -, con la democracia y los individuos redunda además en una fuerte toma de posición normativa y en una crítica aguda a parte del mainstream politológico estadounidense, que incluye a la ciencia política "procedimentalista" y a la teoría democrática circunscripta exclusivamente al análisis del funcionamiento de las reglas del régimen.

En la concepción de O’Donnell, la democracia es también la posibilidad de la autoafirmación a nivel micro. Dado que: a) sólo la democracia produce una red dialógica en la que coexisten una voz vertical - la posibilidad de interpelar al centro afirmando nuestra identidad e intereses sobre cuestiones que consideramos relevantes - y una voz horizontal - que hace posible el asociacionismo, y una voz colectiva compartida; b) para que esa red sea efectivamente democrática debemos tener derecho a hacer esas interpelaciones y gozar de libertades que las protejan; y c) para poder ser nosotros mismos y tener una identidad debemos proyectarla en un espacio simbólico interactivo; entonces sólo la democracia garantiza redes dialógicas que hacen a los seres humanos más completos, realistas y maduros, y a las instituciones democráticas más abiertas, receptivas y responsables ${ }^{3}$. Por otro lado, la concepción del ser humano como agente que O'Donnell, entre otros, ha desarrollado - un ser dotado de razón práctica que puede esperar ser respetado en su dignidad como tal y la provisión social de las condiciones necesarias para ejercer libremente sus derechos - es a la vez el resultado de la convergencia de i) las corrientes actuales sobre derechos humanos y desarrollo humano, que invocan a esa concepción del ser humano desde una mirada universalista pero sin accionabilidad judicial, lo que es contrarrestado por ii) la democracia política, que la invoca sobre la base de la nacionalidad pero por lo tanto de alcance restringido, lo que es contrarrestado por iii) la adopción de convenios internacionales en las leyes domésticas, la extensión de derechos civiles, sociales (y hasta políticos) a todos los habitantes, incluidos residentes no nacionales.

Insisto en el punto, una mirada más atenta de éste y otros autores dedicados a trabajar en el campo de la calidad de la democracia le hubiese

\footnotetext{
${ }^{3}$ Sin embargo, estos procesos se interrumpen cuando el Estado se aliena, se reifica, es decir cuando se pierde de vista su origen y la justificación de su poder y se presenta como un otro situado por encima de los ciudadanos, transformados en meros sujetos - en un proceso análogo a la fetichización del capital y las mercancías en Marx.
} 
permitido a Cansino (2008) observar que para muchos de ellos hay múltiples razones que hacen preferible a la democracia política ${ }^{4}$, pero ella es sólo un aspecto, indispensable, de la democracia. Una adecuada conceptualización de la democracia, requiere para O’Donnell moverse en tres niveles - ciudadanía, régimen y Estado - con conciencia de que cada caso tiene legados históricos y diferencias comparativas. Y como el carácter siempre abierto de la democracia prohíbe una definición rígida, la teoría de la democracia y la política comparada que propone se orientan más a la democratización que a la democracia, es decir, a la adquisición y respaldo legal de derechos y libertades civiles, políticas, sociales y culturales. En otras palabras, una visión de la democracia que tiene en cuenta las condiciones sociales de la ciudadanía, y no sólo la constitución de los "votantes". En estos argumentos residen además, algunas de las polémicas que se han sostenido últimamente (ver O’Donnell, 2011).

\section{La política comparada sobre América Latina}

Una consecuencia de no encarar seriamente los debates internos a la politología es una percepción falsa sobre la relevancia de las distintas subdisciplinas dentro de la disciplina abarcante, la ciencia política. En este sentido, en las universidades latinoamericanas muchas veces se cree que la política comparada ejerce una supuesta hegemonía dentro del campo disciplinar, y más aún, que la política comparada es parte de una avanzada de las supuestamente conservadoras teorías neoinstitucionalistas o de la elección racional. Un examen empírico de la producción académica nos orienta en una dirección diferente.

Para empezar, y a pesar del conocimiento acumulado, la ciencia política es, aún, una disciplina fragmentada conceptual y metodológicamente (ALMOND, 1999; NEGRETTO, 2004; HARTLYN, 2010). En Estados Unidos, si bien es cierto que en los últimos años hay más atención a los métodos cuantitativos y a la formalización de argumentos (mucho más, por otra parte, en los posgrados que en los grados), y que la hiperespecialización metodológica puede justificar investigaciones más por el método que por la importancia real del tema en cuestión, también es cierto que ésta no es una posición compartida por todos los cultores de la disciplina en ese país, ni mucho menos hegemónica. No sólo hay importantes debates en las universidades y en las asociaciones de ciencia política

\footnotetext{
${ }^{4}$ En términos de O’Donnell (2010), la democracia política brinda maneras pacíficas de decidir quién gobernará durante un tiempo; provee el único recurso político igualitario (el voto); permite resolver los conflictos entre derechos y obligaciones de maneras accionables legalmente (es también un sistema de derechos); sus derechos son útiles para democratizar diversos espacios sociales; da igual peso a todos los votantes; las decisiones derivan de procesos ascendentes y se ajustan a las reglas legales; los ciudadanos son la fuente y justificación de la autoridad y de los poderes que emanan de ella; las elecciones des-reifican todos los poderes, sobre todo el del Estado; y sus prácticas son una base para luchar contra las tendencias desigualadoras del capitalismo y las burocracias.
} 
sobre estos temas ${ }^{5}$, sino que los cultores de las metodologías cualitativas son muy importantes ${ }^{6} \mathrm{y}$, reafirmando a la ciencia política como una ciencia que utiliza múltiples métodos - con la mirada puesta en que muchos fenómenos observados por la ciencia política pueden requerir interacciones complejas de varios factores han avanzado en temas sensibles como la medición de conceptos e, incluso, la formalización del análisis de los métodos cualitativos (HARTLYN, 2010).

Otra de las falacias más comunes sostiene que dentro del campo de la política comparada estadounidense y de otros países desarrollados, la política latinoamericana no tiene ninguna incidencia, y por lo tanto ese no es un campo que aquellos interesados en la región debieran frecuentar. Esta es una visión no sólo equivocada, sino también anacrónica. La política comparada de Estados Unidos sobre América Latina ha tenido cuatro dimensiones o focos de atención: a) primero ha sido altamente descriptiva de una zona poco factible de ser comprendida en sus fundamentos culturales desde el centro de la disciplina; b) luego aparecieron trabajos que explicaban con escaso detalle las instituciones políticas y narraban las etapas de su historia, con énfasis en sistemas jurídicos y de gobierno. Después de la Segunda Guerra Mundial se empezó a distinguir entre sus subregiones (Sudamérica, el Caribe, el Cono Sur) pero sin revisar políticas o actores concretos dentro de condiciones sincrónicas o diacrónicas de estudio; c) más tarde se produjeron trabajos de coyuntura asociándolos con macroprocesos como la democracia, la modernización o la revolución; y d) finalmente se escribieron estudios de actores (élites, partidos políticos, movimientos sociales, género, procesos electorales, minorías culturales) o políticas públicas concretas en los que el estudio del Estado siguió siendo primordial, para llegar a estudios de bienes o servicios concretos (educación, criminalidad, violencia social, rendición de cuentas, pobreza, etc.) (ALARCÓN OLGUín, 2011). A pesar de la influencia de los enfoques pioneros y de cierto consenso metodológico en la construcción del campo, en el siglo XXI se han generado preguntas de investigación, técnicas y bancos de datos (incluyendo la comparación subnacional) que responden a un "despegue" endógeno y han dado lugar a importantes puntos de discusión propios (ALARCÓN OLGUÍN, 2011). Para Jonathan Hartlyn (2010), el hecho que gran parte de las universidades más importantes de Estados Unidos tengan centros dedicados al estudio de América Latina es un indicador de que los estudios de política comparada sobre América Latina son una parte vital de la ciencia política de ese país:

\footnotetext{
5 Incluso el movimiento de reforma dentro de la disciplina, llamado Perestroika, es objeto de estudio de un libro: Monroe (2005).

${ }^{6}$ La sección organizada de la American Political Science Association (APSA) más grande después de la de metodología política es la de investigación cualitativa y métodos múltiples, que cuenta además con la revista Qualitative Methods.
} 
A nivel de grado se dictan cursos sobre la política de América Latina y sobre las relaciones entre Estados Unidos y Latinoamérica en todos los principales departamentos de Ciencia Política del país, y con menos frecuencia, también a nivel de posgrado. La gran mayoría de los principales departamentos de Ciencia Política que ofrecen el doctorado tiene profesores en política comparada que enfocan el estudio de América Latina (HARTLYN, 2010, p. 145-146).

Para el resto del mundo, una cita de Alarcón Olguín (2011, p. 159-160) resume a nuestro criterio muy bien los aportes principales:

Desde la experiencia europea, la presencia desde España de la revista América Latina Hoy y la Universidad de Salamanca han tomado un liderazgo importante, si bien ello no minimiza trabajos de difusión como lo que hicieron la Universidad Complutense o el Instituto Ortega y Gasset durante décadas. La aportación inglesa no ha sido menor desde sus universidades más importantes como Essex, Oxford y Cambridge, cuyas editoriales son una de las fuentes de novedad constante en la publicación de temas comparativos y latinoamericanos, ni que decir el papel jugado por investigadores como Laurence Whitehead, George Phillip o Alan Knight, quienes han sido baluartes importantes para mantener el interés por la región. En los Estados Unidos, el trabajo de universidades y 'think tanks' como Notre Dame, Harvard, Chicago, Columbia, Texas-Austin, el Woodrow Wilson Center, Georgetown, Berkeley, San Diego, Stanford, Tulane, Vanderbilt, Pittsburgh, entre muchas otras, se complementa con la labor importante de editoriales e importantes redes de trabajo que han tenido sobre todo en LASA a su más importante promotor a partir de los congresos que regularmente se realizan desde hace más de 40 años. En Francia, la presencia de una revista simbólica como los Cahiers des Amériques Latines, así como gente como Alain Touraine, Georges Couffignal, Alain Rouquié y más recientemente, Olivier Dabéne o Hélene Combes mantienen vivo el interés en dicho país. En Alemania, los trabajos hechos por Dieter Nohlen en Heidelberg, o el GIGA de Hamburgo, bajo la batuta de Detlef Nolte y Klaus Bodemer, señalan una clara comunicación y presencia en la región latinoamericana. Ello no desmerece el interés que existe desde espacios como Holanda, Italia, Suecia, Austria e incluso Rusia. El Congreso de Americanistas en Europa promovido por CEISAL también ha sido por largo tiempo uno de los espacios primordiales para el conocimiento de la situación latinoamericana en dichas regiones.

Que la región latinoamericana ha mostrado en las últimas dos décadas ser una fuente de conocimiento importante para los comparativistas (sobre diversos aspectos de la política y también, afortunadamente, sobre el funcionamiento de la democracia) también puede ser demostrado a través de análisis bibliométricos. El 
estudio de las tres publicaciones más importantes en el área ${ }^{7}$ realizado por Munck y Snyder (2006) muestra que hay una presencia importante de trabajos sobre América Latina: el $27 \%$ en el período 1989-2004, sólo precedido por un $41 \%$ sobre Europa occidental ${ }^{8}$. Por su parte, Hartlyn (2010) revisó la presencia de especialistas en América Latina en nueve revistas estadounidenses de ciencia política entre 2001 y $2005^{9}$ y encontró que en todas ellas hay especialistas sobre América Latina: 19 artículos en las revistas generales, 87 en las de política comparada, y 112 en las latinoamericanistas ${ }^{10}$. Si bien podría argumentarse que las exigencias de productividad hacen que en Estados Unidos se publique mucho, y que por tal motivo los autores prefieren la seguridad de los resultados publicables antes que el riesgo intelectual, la presencia de la región en la agenda de la política comparada no deja de ser un dato relevante.

Resulta pertinente destacar, junto con Rossana Castiglioni (2011) que en casi todos los campos en los que la política comparada ha centrado su atención en América Latina (en el estudio de los quiebres democráticos, el surgimiento de regímenes autoritarios, los procesos de transición y democratización, consolidación, y más recientemente, el desempeño o la calidad de la democracia) Guillermo O’Donnell fue uno de los autores que más contribuyó a ubicar a la región en el centro de la agenda.

\section{La política comparada en América Latina y Argentina}

La política comparada es tanto un área temática como una metodología. En palabras de Urbani (1997), implica qué cosa comparar y al mismo tiempo cómo comparar. En palabras de Mair (2001), la política comparada se define tanto por su sustancia (el estudio de una pluralidad de países) como por su método, pero ambos combinados simultáneamente - dado que la sustancia sola no se diferencia de la ciencia política como un todo, y el método solo es aplicable a todas las ciencias sociales. Con respecto al método no nos concierne aquí decir mucho ${ }^{11}$.

\footnotetext{
${ }^{7}$ Comparative Political Studies, Comparative Politics y World Politics.

${ }^{8}$ Sin embargo, apenas algo más del $1 \%$ de los autores que han publicado en esas revistas en ese mismo período residen en la región. Esto marca claramente que, al menos en la investigación de punta, la política comparada latinoamericana necesita todavía de muchos avances de todo tipo.

${ }^{9}$ Unas de carácter general: American Political Science Review, Journal of Politics y American Journal of Political Science; otras específicas de política comparada: Comparative Politics, Comparative Political Studies, World Politics y Studies in Comparative International Development; y otras sobre América Latina: Latin American Politics \& Society y Latin American Research Review.

${ }^{10}$ Sin embargo, en las revistas generales de ciencia política, donde reside el mayor prestigio profesional, los artículos publicados sobre la región son limitados: sólo el 7,7\% en promedio en las seis principales revistas de Estados Unidos (American Political Science Review, American Journal of Political Science, Journal of Politics, World Politics, Comparative Political Studies y Comparative Politics) (PÉREZ-LIÑÁN, 2010).

${ }^{11}$ Una muy buena sistematización es Morlino (2005).
} 
En cuanto a la sustancia, o en otras palabras, a la agenda de investigación, también aquí el debate está mal orientado.

Nuevamente, la comparación con Estados Unidos y otros países desarrollados es a la vez ilustrativa pero relativa. Como hemos visto, en términos generales el debate que se ha suscitado en el centro de la ciencia política mundial $^{12}$, y de la política comparada en particular - si bien hay una gran discrepancia en el campo específico de la política comparada (ver Munck y Snyder, 2007) - gira alrededor del avance del rational choice y de los modelos formales que simplifican demasiado la realidad (SARTORI, 2005; SCHMITTER, 2009). Sin embargo, el auge del rational choice y la teorización con modelos lógicos matemáticos están a años luz de nuestra realidad regional. Si bien hay algunos cultores, esta escuela es muy minoritaria dado que en la región suele haber poca formación metodológica (sobre todo en análisis matemático). De un lado, esto tiene la ventaja de evitar la concentración en el método (y sus consecuencias en la creación de teorías positivas) restando importancia a los problemas políticos reales (GREEN y SHAPIRO, 1994; SHAPIRO, 2002). Pero por el otro, se multiplica exponencialmente el riesgo de caer en las narrativas y el ensayismo coyuntural y superficial. La práctica (y la enseñanza) de la política comparada requieren de saberes teóricos, históricos y metodológicos previos, pero es en este último punto donde se encuentran las mayores deficiencias (BULCOURF, 2008). En nuestro medio, toda vez que pueda escaparse a las más inmediatas y hasta provocativas impugnaciones, en términos de Almond (1999) desde la "izquierda blanda"13, volcarse por una determinada pregunta de investigación, un determinado enfoque teórico o un determinado método deben tomarse más como una saludable muestra del pluralismo teórico y metodológico que como una toma de posiciones en una guerra de trincheras. Como sostienen Lodola y Saiegh,

[...] la cuestión, entonces, no se reduce a una simple batalla entre irreconciliables fundamentalismos metodológicos. Muy por el contrario, lo importante es determinar la utilidad práctica de cada método y establecer las condiciones bajo las cuales estos pueden complementarse (LODOLA y SAIEGH, 2004, p. 28).

¿Cuán "hegemónica" es la política comparada en América Latina? A pesar de los importantes avances en las últimas dos décadas ${ }^{14}$, una mirada rigurosa a la producción politológica regional puede ser reveladora. Para contestar la pregunta,

\footnotetext{
${ }^{12}$ El 75\% de los politólogos del mundo son estadounidenses (MARSH y SAVIGNY, 2004).

13 Aún en las revistas estadounidenses paradigmáticas, las investigaciones de política comparada sobre la región son mayormente cualitativas y muy poco formalizadas (MUNCK y SNYDER, 2006).

${ }^{14}$ Por ejemplo, en 2008 ha salido en una Revista Latinoamericana de Política Comparada editada por el Centro Latinoamericano de Estudios Políticos (CELAEP), con sede en Ecuador, pero es un proyecto algo reciente - apenas ha editado tres números - y esporádico.
} 
entonces, los análisis bibliométricos han sido los preferidos de varios investigadores. Fanelli y Di Filippo (2011) analizaron cuatro revistas de ciencia política de la región ${ }^{15}$ entre 2003 y 2007 , y encontraron que es minoritaria la publicación de artículos de política comparada (29\%) y bastante alto el nivel de parroquialismo. Stumpf González (2011) analizó seis revistas brasileñas de ciencia política $^{16}$ entre 2006 y 2011, y encontró que sólo el 7,5\% de los artículos publicados son comparativos, porcentaje que no está en crecimiento. Además, descubrió que de las 955 tesis de diez posgrados en ciencia política defendidas entre 1985 y 2000 en Brasil, solamente el 3\% usó el método comparado, y ninguna de ellas datos cuantitativos. También sobre el caso brasileño, Marenco (2011) examinó sólo dos de los journals científicos ${ }^{17}$ entre 2006 y 2011 para sostener que dos terceras partes son estudios de caso (abrumadoramente, el de Brasil), que menos del $4 \%$ usan un $\mathrm{N}$ elevado, y menos de un $15 \%$ son artículos comparados, entre los cuales los temas predominantes son el presidencialismo y el poder legislativo. Por su parte, Lucca y Puello-Socarrás (2011) analizaron tres revistas colombianas ${ }^{18}$ entre 1985 y 2010. Allí los artículos de política comparada no llegan al $10 \%$. De ellos, el $40 \%$ se refiere a temas concernientes a procesos políticos, electorales, institucionales y democracia. También examinaron cuatro revistas mexicanas ${ }^{19}$ entre 2000 y 2010 y hallaron que los artículos politológicos comparativos son el 19\%, la mayor parte de los cuales son estudios de un caso (generalmente el mexicano) para ilustrar un argumento o teoría comparativos. De ellos, los temas más frecuentes son el comportamiento electoral y los partidos políticos (35\%). Finalmente, Chasquetti (2010) ha estipulado que los artículos de política comparada en la Revista Uruguaya de Ciencia Política entre 1987 y 2008 representan sólo el $20 \%$ del total, dado que en esa publicación hay un claro dominio de los estudios de caso (ver también Rocha, 2012). Esta y otras circunstancias han llevado a este autor a sostener que la utilización del método comparado en la región es insuficiente, y que la formulación y el control de hipótesis para un único caso "transforma a la ciencia política en una disciplina fragmentada y con una débil pretensión de generalización teórica" (CHASQUETTI, 2011, p. 1)

En Argentina la situación no es más alentadora para la política comparada $^{20}$. Teniendo en cuenta el desarrollo nacional de la subdisciplina, la

\footnotetext{
${ }^{15}$ Revista Uruguaya de Ciencia Política, Revista de Ciencia Política (Chile), Sociología y Política (Brasil) y Revista Brasileira de Ciências Sociais.

${ }^{16}$ Dados, Opinião Pública, Brazilian Political Science Review, Revista Brasileira de Ciências Sociais, Revista de Sociologia e Política y Debates.

${ }^{17}$ Dados y Opinião Pública.

${ }^{18}$ Colombia internacional, Análisis político y Papel político.

19 Política y gobierno, Perfiles latinoamericanos, Revista Mexicana de Ciencias Políticas y Sociales y Polis.

${ }^{20}$ Dado que Argentina es uno de los casos de mayor desarrollo (junto con Brasil y México) de la ciencia política dentro de América Latina (ALTMAN, 2006; HUNEEUS, 2006) y que la política
} 
llegada de la democracia en 1983 significó un verdadero cambio de paradigma en la política comparada en tanto se fue abandonando la mirada estrictamente jurídica de los sistemas políticos hacia la consideración relevante de las cuestiones metodológicas (GEARY, ORTA y ARMENDÁRIZ, 2011). Sin embargo, en nuestros días, la influencia de la política comparada en Argentina es relativa. Más allá del loable portal <politicacomparada.com.ar>, que ha editado seis buenos boletines, no existen en Argentina revistas específicas de política comparada ni tampoco bibliografía sobre metodología comparada de producción nacional. Jolías y Reina (2009) relevaron casi 700 artículos publicados en siete revistas de ciencia política de Argentina entre 1996 y $2008^{21}$, y encontraron que sólo el $16 \%$ de ellos es de política comparada. De ellos, apenas la mitad fueron escritos por autores argentinos. Perbellini y Pinillos (2011) estudiaron cuatro revistas relevantes en el campo de la política comparada en el país ${ }^{22}$ entre 2000 y 2010, y encontraron que la mayor parte $(56 \%)$ de los artículos publicados pertenecientes al campo de la política comparada fueron escritos por autores filiados en instituciones académicas de otros países. Entre los temas más presentes figuran los partidos y los sistemas de partidos (25\%), el presidencialismo (20\%), las elecciones y los sistemas electorales (10\%), la calidad de la democracia (10\%) y comparaciones de políticas públicas (10\%). En cuanto a los casos estudiados por los artículos, sobresalen los latinoamericanos (86\%). La mayoría de estos artículos $(48 \%)$ son estudios de caso que se enmarcan en una perspectiva comparada, luego los estudios de tres a 20 casos (30\%) y luego los estudios de dos casos (16\%).

El análisis bibliométrico de las revistas locales es útil para sacar conclusiones generales. En las ciencias sociales argentinas suele ser bastante escasa la internacionalización de la producción académica. Gantman (2011) estudió los CVs de (entre otros científicos sociales) 83 investigadores del Conicet en ciencia política y Relaciones Internacionales. Allí predominan los artículos en publicaciones locales (casi 60\% del total), luego los capítulos de libros en el país, registrándose una proporción muy baja de artículos en revistas en inglés (casi 3\% del total). En comparación con otras disciplinas, la internacionalización de la producción de los politólogos (relacionada estadísticamente a la poca cantidad de doctorados en otros países) es inferior a la de los economistas, los sociólogos y los psicólogos.

En cuanto a la producción presentada en reuniones científicas, los mayores eventos de la disciplina (los congresos nacionales de ciencia política organizados por la entidad que nuclea a los politólogos a nivel nacional, la Sociedad Argentina

comparada requiere de cierta sofisticación teórica y metodológica dentro del gran conjunto de la ciencia política, estimamos que la situación en la gran mayoría de los países de los que no conocemos datos es peor.

${ }^{21}$ Revista Argentina de Ciencia Política, POSTData, Revista SAAP, Política y Gestión, Desarrollo Económico, El Debate Político y Revista de Ciencias Sociales (UNQ).

${ }^{22}$ Revista SAAP, POSTData, Desarrollo Económico y Temas y Debates. 
de Análisis Político, SAAP) incorporaron paneles específicos de política comparada recién en 2003 (JOLÍAS y REINA, 2009). A partir de esa fecha, a pesar de que han ido creciendo paulatinamente, las ponencias de política comparada representan un 11,9\% (ver Tabla 1).

Tabla 1 - Ponencias de política comparada (PC) en los congresos nacionales SAAP (2003-2011)

\begin{tabular}{c|c|c|c|c|c|c}
\hline & $\begin{array}{c}\text { VI } \\
\text { Congreso } \\
(\mathbf{2 0 0 3 )}\end{array}$ & $\begin{array}{c}\text { VII } \\
\text { Congreso } \\
\mathbf{( 2 0 0 5 )}\end{array}$ & $\begin{array}{c}\text { VIII } \\
\text { Congreso } \\
\mathbf{( 2 0 0 7 )}\end{array}$ & $\begin{array}{c}\text { IX } \\
\text { Congreso } \\
\mathbf{( 2 0 0 9 )}\end{array}$ & $\begin{array}{c}\mathbf{X} \\
\text { Congreso } \\
\mathbf{( 2 0 1 1 )}\end{array}$ & Total \\
\hline Total ponencias & 239 & 263 & 208 & 521 & 1074 & 2305 \\
\hline Ponencias de PC & 22 & 26 & 21 & 70 & 186 & 325 \\
\hline Promedio & $9,2 \%$ & $9,8 \%$ & $10 \%$ & $13,4 \%$ & $17,3 \%$ & $11,9 \%$ \\
\hline
\end{tabular}

Fuente: Elaboración propia y De Luca (2006), Jolías y Reina (2009) y Rotman (2010 y 2012).

A pesar de lo anterior, en el lado del debe podría señalarse la existencia de un "núcleo duro" en el plano de la enseñanza: la asignatura Política Comparada (o denominación similar, generalmente Sistemas Políticos Comparados) está presente en la gran mayoría de los planes de estudio de las licenciaturas en ciencia política. A diferencia de otras materias (como las materias de Introducción a la Ciencia Política, o sus similares) presenta un grado importante de homogeneidad en cuanto a temas (y autores) abordados: el método comparado (los autores más presentes son Sartori, Bartolini, Collier, Morlino, Lijphart y Almond), los regímenes políticos y la democratización (Collier, Morlino, Dahl, Linz, Pasquino, Tsebelis, Lijphart y Mainwaring), las formas de gobierno (Linz, Sartori, Mainwaring, Lijphart y Tsebelis) partidos y sistemas de partidos (Sartori, Pasquino, Mainwaring, Panebianco y Lipset) y sistemas electorales (Sartori, Lijphart, Colomer y Cox). Entre los autores latinoamericanos, el de mayor presencia es, lógicamente, Guillermo O'Donnell, seguido por Gerardo Munck, Aníbal Pérez Liñán y Jorge Lanzaro ${ }^{23}$. En un trabajo más exhaustivo, Geary, Pinillos, Lucca y Borrell (2010) relevaron 23 programas de materias de grado en Argentina de política comparada entre 2002 y 2007. En general, la política comparada es abordada por la temática y su estudio a la luz de casos (formas de gobierno, sistemas de partido, tipos de democracia, etc.) y en mucho menor medida por su método. Sartori, Lijphart y Mainwaring y Shugart están presentes en el $80 \%$ de los programas, mientras que Collier, Linz, Panebianco, O’Donnell y Pasquino están presentes en el $50 \%$ de los programas. Esta homogeneidad es saludable desde el punto de vista de una base de conocimiento común y compartida, pero también puede ser un signo de estancamiento. En los centros

\footnotetext{
${ }^{23}$ Datos recolectados de ocho programas de materias de política comparada de las universidades de Buenos Aires, del Salvador, de San Andrés, Nacional de Entre Ríos, Nacional de La Matanza, Nacional de San Martín y Católica de Santa Fe.
} 
mundiales de la política comparada, lo que se enseña en las aulas cambia con una velocidad mucho mayor debido a la mayor productividad, la cual, a su vez, es producto de la especialización y la creación de redes, y del consecuente aumento de la colaboración en la autoría de los trabajos. Así, hoy es muy difícil que un politólogo bien formado pueda dominar aceptablemente todas las áreas de la disciplina. En cambio, en esos centros, es bastante común que los expertos en psicología política, por ejemplo, colaboren con los expertos en modelos matemáticos, con los expertos en estadística y con los expertos en instituciones para escribir juntos un artículo sobre algún tema que toca esas subáreas.

En cuanto a los temas de investigación futura de la política comparada regional, no los conocemos bien, porque en general están determinados por la política nacional (NOHLEN, 2006). A pesar de que en Argentina - y esto pareciera ser relativamente excepcional (ver el vol. 25, n. 1 de la Revista de Ciencia Política, PUCCh) - la agenda temática ha dependido en gran parte de la agenda de investigación de Estados Unidos, la principal variable independiente han sido las prioridades de los tomadores de decisiones: en los 1980 la "consolidación" de la democracia, la atenuación del presidencialismo y la eficacia y eficiencia de la administración pública; en los 1990 las tendencias delegativas, la relación entre el Poder Ejecutivo y el Poder Legislativo, el proceso de reformas económicas y las políticas sociales; en los 2000 la crisis de representación y de los partidos políticos, la protesta social y la relevancia de la política subnacional (LEIRAS, ABAL MEDINA y D'ALESSANDRO, 2005; TORRE, 2007).

Para la producción y la transmisión del conocimiento científico (en nuestro interés aquí, para hacer política comparada) es también indispensable lo que podríamos denominar la "infraestructura" de la investigación y la docencia. Es ya bien conocida la escasez de recursos de todo tipo en la inmensa mayoría de las universidades de la región, sobre todo en el ámbito de las ciencias sociales, y particularmente en el de la ciencia política. La política comparada es muy costosa comparada con otras subdisciplinas de la ciencia política, y en general carecemos de la infraestructura requerida para proyectos comparativos (fondos para equipamiento de todo tipo, viajes, estadías largas, para no mencionar bibliotecas, oficinas, aulas u otros espacios de trabajo). Este escaso desarrollo institucional y académico es la principal causa por la cual emigran, principalmente a Estados Unidos, los colegas de la región que quieren estudiar y/o hacer política comparada en mejores condiciones. En este punto Brasil goza de cierta ventaja: los brasileños emigran menos - los posgrados brasileños son de mejor calidad, son más competitivos internacionalmente, y tienen mejores políticas de financiamiento por parte del Estado - y/o regresan más a su propio país que los argentinos y los uruguayos (FREIDENBERG y MALAMUD, 2013). Algo similar ocurre en el ámbito de las relaciones internacionales (TICKNER, CEPEDA y BERNAL, 2012). 
Como una consecuencia de lo anterior, se produce una mayor fragmentación de las comunidades politológicas locales. En su célebre libro Modernización y autoritarismo, Guillermo O'Donnell (1972) argumenta que en los procesos de modernización de algunas sociedades latinoamericanas, la importancia y el impacto de los roles tecnocráticos que ejercen algunos individuos (que se gradúan en el exterior o en escuelas técnicas con planes transplantados o copiados del exterior) no reside tanto en los saberes específicos que obtienen sino precisamente en los modelos de roles que absorben y luego transmiten en sus sociedades originales. Estos roles generan un "transplante" de expectativas, vinculados a diferentes criterios en cuanto a logros, el tipo de apoyos institucionales y recompensas esperados en el desempeño de dichos roles, los parámetros para la elección de las técnicas adecuadas o los criterios de eficiencia, etc. Análogamente, en los procesos de modernización de las esferas académicas de nuestros países pareciera ocurrir un fenómeno similar. En ocasiones estos roles más modernos, absorbidos en los estudios de posgrado en el exterior, al querer transplantar prácticas académicas provenientes de contextos educativos y científicos más avanzados, desconocen o no logran descifrar de manera adecuada el contexto local, que termina frustrándolos y aislándolos en comunidades de reconocimiento mutuo cada vez más endogámico. Ello genera una mayor diferenciación hacia el interior de la comunidad en lugar de la modernización académica a través de la integración de los diferentes grupos, lo cual a la postre resulta de muy escasa utilidad académica para el desarrollo de una disciplina científica y consecuentemente para la sociedad en su conjunto. Por supuesto, los extremos deberían evitarse: tanto el transplante acrítico como la resistencia a la modernización en nombre de las particularidades locales generan efectos negativos sobre la comunidad académica que no hacen otra cosa que reforzar las debilidades locales y fragmentar aún más la por naturaleza heterogénea composición del ámbito politológico.

Todo ello redunda, finalmente, en la persistencia del multiempleo en los comparativistas latinoamericanos (ALTMAN, 2006), y como corolario del poco financiamiento, posgrados con problemas de todo tipo. No sólo casi no hay centros de investigación de política comparada (en Argentina no hay ninguno) sino que hay además pocas redes de proyectos co-gestionados. Ha habido en este sentido algunas experiencias, pero que no se han traducido en análisis comparativos de carácter sistemático (TORRE, 2007) o sostenidos en el tiempo ${ }^{24}$.

Por último, en las universidades en las que se gradúa la mayor cantidad de politólogos del país (y de otros países, como Uruguay, por ejemplo), hay condiciones particulares que hacen muy difícil mejorar la situación de la política comparada. En Argentina la Universidad de Buenos Aires (y otras grandes, como

\footnotetext{
${ }^{24}$ Una de estas excepciones fue la cooperación de varios politólogos que hacían estudios de opinión en los años 1980, y que diera lugar a lo que luego fue Latinobarómetro (HUNEEUS, 2006).
} 
la Universidad Nacional de Rosario) tienen carreras que escapan a las definiciones más formales de lo que es (o debería ser) un departamento académico: lejos de ser grupos de profesores que trabajan juntos dentro de los límites de una disciplina con objetivos compartidos (ALTMAN, 2012) son amplios grupos de profesores también son relevantes los graduados y las agrupaciones estudiantiles enfrentados políticamente, y además con visiones contrapuestas, y en pugna, sobre lo que es o debería ser la propia disciplina. En estos contextos de "colonización de la ciencia política" y que han dado lugar a un "vaciamiento del centro teóricodisciplinar" (BULCOURF y CARDOZO, 2011), la política comparada goza de poca centralidad en la disciplina y por tanto en los planes de estudio, lo cual retroalimenta un círculo vicioso de irrelevancia y falta de recursos de todo tipo: profesores, dedicaciones docentes de tiempo completo, proyectos de investigación, becas, tesis, docentes auxiliares, publicaciones, prestigio, etc. Como corolario, las universidades de América Latina en general, y de Argentina en particular, no tienen producción ni transmisión significativas de política comparada, por lo que el conocimiento que se alienta tiende a promover explicaciones parroquiales (GEARY, PINILLOS, LUCCA y BORRELL, 2010) por parte de ensayistas críticos de la realidad local que, a fin de cuentas, se transforman en productores de materias primas (estudios de casos) que delegan el análisis comparativo/global al centro estadounidense.

\section{El aporte al debate público}

Para finalizar, una nota sobre la incidencia pública de la política comparada. Quizás aquí quepa el comentario más optimista. Philippe Schmitter (2009) ha sostenido que la sofisticación teórica y metodológica contribuye a que los comparativistas, en especial los estadounidenses, sólo pretendan ser escuchados por sus colegas. Sin embargo, en la realidad regional, los politólogos en general están teniendo una creciente intervención en el debate político público (TORRE, 2007), y mucho más en los últimos cinco años. Ello es un dato positivo porque el desarrollo de la política comparada puede favorecer el conocimiento social de los fenómenos políticos, así como los comportamientos políticos de los ciudadanos. En palabras de Urbani (1997, p. 1229): 


\begin{abstract}
[...] es evidente la vinculación existente entre los progresos realizados por el conocimiento político en materia científica y las oportunidades de maduración cívica que derivan de los mismos en el nivel de los comportamientos políticos cotidianos: mejorar los propios conocimientos conduce, por lo menos, a actuar con un dominio cada vez mayor de los propios actos. Pero ése no es el único resultado. Comparar no es sólo un procedimiento científico; es también un modo de pensar a través del cual aumentan las posibilidades de 'aprender de los demás', atesorando las lecciones que vienen de la experiencia de sistemas políticos diferentes del nuestro. Comparar, en este sentido, quiere decir poder disminuir los riesgos de la experimentación en la oscuridad: una contribución de incalculable importancia, por lo tanto, para el estadista que debe decidir y para el ciudadano que es llamado a juzgar a quien lo gobierna.
\end{abstract}

Siempre será bueno recordar, sin embargo, que la política comparada no resuelve problemas políticos, más bien los identifica y, sobre todo, su utilidad radica consecuentemente en brindar un lenguaje y un debate acorde con ello, es decir, brindar marcos analíticos y herramientas conceptuales precisas (SCHEDLER, 2004). A fin de cuentas, el debate público cambia de acuerdo a cómo se interpreten algunos términos típicos de la política comparada: no es lo mismo "guerra" que "terrorismo", "revolución" que "transición", "democracia delegativa" que "populismo", o "partido hegemónico" que "partido predominante". Ahora bien, y más importante aún, la precisión debe ser mayor en cuestiones relativas a la ingeniería constitucional comparada: probablemente, la intervención y el aporte de los comparativistas sea crucial en Argentina si es que prospera la discusión (hasta ahora también muy mal orientada) sobre las supuestas ventajas del parlamentarismo sobre el presidencialismo, o de las ventajas de la concentración del poder sobre la separación de poderes.

\title{
Martín D'Alessandro é Professor de Ciência Política da Universidade de Buenos Aires e investigador do Conselho Nacional de Investigações Científicas e Técnicas (CONICET) da Argentina e do Instituto de Investigações "Gino Germani" da Universidade de Buenos Aires. E também é diretor das Revistas de Ciência Política POSTData e Revista SAAP. E-mail: martindalessa@gmail.com
}

\section{Referencias}

ALARCÓN OLGUÍN, Víctor. La política comparada sobre y desde América Latina (notas para una agenda de estudio). In: GEARY, Mirta; LUCCA, Juan Bautista; PINILLOS, Cintia (comps.). Tendencias en la producción del 
conocimiento sobre política comparada en América Latina. Rosario: Ediciones del Revés, 2011. p. 153-170.

ALMOND, Gabriel A. Mesas separadas: escuelas y corrientes en las ciencias políticas”. In: ALMOND, Gabriel A. Una disciplina segmentada. Escuelas y corrientes en las ciencias políticas. México D.F.: FCE, 1999. p. 39-62.

ALTMAN, David. From Fukuoka to Santiago: Institutionalization of Political Science in Latin America. Political Science and Politics, Washington, v. 39, n. 1, p. 196-203, enero, 2006.

Where is Knowledge Generated? On the Productivity and Impact of Political Science Departments in Latin America. European Political Science, v. 11, p. 71-87, enero, 2012.

BULCOURF, Pablo. Enseñar a comparar: algunos desafíos de la enseñanza de la política comparada en la Argentina. Boletín de Política Comparada, Buenos Aires, n. 1, p. 59- 80, junio, 2008.

BULCOURF, Pablo; CARDOZO, Nelson. La fábrica de politólogos: la enseñanza de la ciencia política en la Argentina actual. Espacios Políticos, Buenos Aires, a. 12, n. 7, p. 30-33, 2011.

CANSINO, César. La muerte de la ciencia política. Buenos Aires: Sudamericana, 2008.

CASTIGLIONI, Rossana. La política comparada en América Latina: algunas reflexiones generales. In: Jornada de debate sobre la Política Comparada en América Latina, Rosario, 2011.

CHASQUETTI, Daniel. El lento camino de la política comparada en Uruguay. In: Red Latinoamericana de Ciencia Política - ReLaCiP. La enseñanza de la ciencia política en las universidades de América Latina. Rosario: Editorial Ciudad Gótica, 2010. p. 103-118.

Una explicación sobre la supervivencia de la política comparada en la región. In: Jornada de debate sobre la Política Comparada en América Latina, Rosario, 2011.

COLOMER, Josep M. La ciencia política va hacia adelante (por meandros tortuosos). Un comentario a Giovanni Sartori. Política y gobierno, México, v. 11, n. 2, CIDE, segundo semestre, 2004.

D'ALESSANDRO, Martín. Sobre la democracia, la agencia y el Estado. Algunas notas a partir de la teorización de Guillermo O'Donnell. PolHis, Mar del Plata, n. 8, p. 249-257, segundo semestre, 2011.

DE LUCA, Miguel. Ciencia política en Córdoba capital: un balance sobre el VII Congreso Nacional de la SAAP. Revista SAAP, Buenos Aires, v. 2, n. 3, p. 637647, ago. 2006.

FANELLI, Lucrecia; DI FILIPPO, Marilé. Algunas reflexiones críticas sobre la política comparada. Un análisis desde la producción en revistas académicas en América Latina. In: GEARY, Mirta; LUCCA, Juan Bautista; PINILLOS, 
Cintia (comps.). Tendencias en la producción del conocimiento sobre política comparada en América Latina. Rosario: Ediciones del Revés, 2011. p. 97-124.

FREIDENBERG, Flavia; MALAMUD, Andrés. Politólogos on the Run: Contrasting Paths to Internationalization of Southern Cone Political Scientists. Latin American Politics and Society, v. 55, n. 1, p. 1-21, 2013.

GANTMAN, Ernesto R. La productividad científica argentina en Ciencias Sociales: Economía, Psicología, Sociología y Ciencia Política en el CONICET (2004-2008). Revista Española de Documentación Científica, Madrid, v. 34, n. 3, jul.-sep. 2011.

GEARY, Mirta; PINILLOS, Cintia; LUCCA, Juan Bautista; BORRELL, Mariana. ¿Qué, cómo y para qué enseñamos política comparada en las aulas argentinas? In: Red Latinoamericana de Ciencia Política - ReLaCiP. La enseñanza de la ciencia política en las universidades de América Latina. Rosario: Editorial Ciudad Gótica, 2010, p. 119-136.

GEARY, Mirta; ORTA, Melisa; ARMENDÁRIZ, Juliana. El desarrollo de la ciencia política en Argentina y su corolario en la enseñanza de política comparada en la Universidad Nacional de Rosario. In: GEARY, Mirta; LUCCA, Juan Bautista; PINILLOS, Cintia (comps.). Tendencias en la producción del conocimiento sobre política comparada en América Latina. Rosario: Ediciones del Revés, 2011. p. 23-56.

GREEN, Donald; SHAPIRO, Ian. Pathologies of Rational Choice Theory: A Critique of Applications in Political Science. New Haven: Yale University Press, 1994.

HARTLYN, Jonathan. La Ciencia Política y el estudio de la política comparada en los Estados Unidos: tendencias y diálogos con la Ciencia Política en América Latina. Anuario Americanista Europeo, n. 8, p. 144-156, 2010.

HUNEEUS, Carlos. El lento y tardío desarrollo de la ciencia política en América Latina, 1966-2006. Estudios Internacionales, Caracas, n. 155, p. 137-156, oct.dic. 2006.

JOLÍAS, Lucas; REINA, Augusto. Las comparaciones no son odiosas. Métodos y estrategias en política comparada. Revista Argentina de Ciencia Política, Buenos Aires, n. 11-12, p. 143-169, jun. 2009.

LAITIN, David D. ¿Adónde va la ciencia política? Reflexiones sobre la afirmación del profesor Sartori de que "la ciencia política estadounidense no va a ningún lado". Política y gobierno, México, v. 11, n. 2, p. 361-367, segundo semestre, 2004.

LEIRAS, Marcelo; ABAL MEDINA, Juan; D'ALESSANDRO, Martín. La ciencia política en Argentina: el camino de la institucionalización dentro y fuera de las aulas universitarias. Revista de Ciencia Política, Santiago, v. 25, n. 1, p. 76-91, 2005. 
LODOLA, Germán; SAIEGH, Sebastián. En defensa del pluralismo y el oportunismo metodológico en política comparada. ¿Ganar una batalla o ganar la guerra? Lo Que Vendrá, Buenos Aires, a. 1, n. 2, p. 28-34, nov. 2004.

LUCCA, Juan Bautista; PUELLO-SOCARRÁS, José Francisco. El alcance de la comparación en la ciencia política colombiana y mexicana. In: GEARY, Mirta; LUCCA, Juan Bautista; PINILlOS, Cintia (comps.). Tendencias en la producción del conocimiento sobre política comparada en América Latina. Rosario: Ediciones del Revés, 2011. p. 125-150.

MAIR, Peter. Política comparada: una visión general. In: GOODIN, Robert E.; KLINGEMANN, Hans-Dieter (eds.). Nuevo manual de ciencia política. Madrid: ISTMO. 2001. p. 447-484.

MARENCO, André. Uma nota sobre usos e não usos de comparaçao. In: GEARY, Mirta; LUCCA, Juan Bautista; PINILLOS, Cintia (comps.). Tendencias en la producción del conocimiento sobre política comparada en América Latina. Rosario: Ediciones del Revés, 2011. p. 163-170.

MARSH, David; SAVIGNY, Heather. Political Science as a Broad Church: The Search for a Pluralist Discipline. Politics, v. 24, n. 3, p. 155-168, 2004.

MONROE, Kristen Renwick. Perestroika!: The Raucous Rebellion in Political Science. New Haven: Yale University Press, 2005.

MORLINO, Leonardo. Introducción a la investigación comparada. Madrid: Alianza, 2005.

MUNCK Gerardo L.; SNYDER, Richard. Debating the Direction of Comparative Politics: An Analysis of Leading Journals. Working Paper, n. 7, p. 1-44, IPSA, marzo, 2006.

. Passion, Craft, and Method in Comparative Politics. Baltimore: The Johns Hopkins University Press, 2007.

NEGRETTO, Gabriel L. Nota del editor. El rumbo de la ciencia política. Política y gobierno, México, CIDE, v. XI, n. 2, p. 347-348, segundo semestre 2004.

NOHLEN, Dieter. Ciencia Política en América Latina. In: NOHLEN, Dieter (ed.). Diccionario de Ciencia Política. México: Porrúa, 2006.

O’DONNELL, Guillermo. Modernización y autoritarismo. Buenos Aires: Paidós, 1972.

Democracia, agencia y estado. Teoría con intención comparativa. Buenos Aires: Prometeo, 2010.

. La retrospectiva de Schmitter: algunas notas de desacuerdo. POSTData, Buenos Aires, v. 16, n. 1, p. 11-25, abr. 2011.

PERBELLINI, Melina; PINILLOS, Cintia. Producción académica y política comparada en Argentina, 2000-2010. In: GEARY, Mirta; LUCCA, Juan Bautista; PINILLOS, Cintia (comps.). Tendencias en la producción del conocimiento sobre política comparada en América Latina. Rosario: Ediciones del Revés, 2011. p. 79-96. 
PÉREZ LIÑÁN, Aníbal. Temas de investigación y perspectivas de la ciencia política latinoamericanista en Estados Unidos. Ponencia presentada en el seminario. El estado de la ciencia política en América Latina: desafíos y oportunidades de la docencia y la investigación en perspectiva comparada, Fundación Global Democracia y Desarrollo, República Dominicana, 27 de enero, 2010.

ROCHA, Cecilia. La ciencia política en Uruguay (1989-2009): temas, teorías y metodologías. Revista Uruguaya de Ciencia Política, Montevideo, v. 21, n. 2, p. 97-127, jul. 2012.

ROTMAN, Santiago. Los logros de la ciencia política argentina. El IX Congreso Nacional de la Sociedad Argentina de Análisis Político en Santa Fe. Revista SAAP, Buenos Aires, v. 4, n. 2, p. 261-270, nov. 2010.

. La promesa de la ciencia política. El X Congreso Nacional de la Sociedad Argentina de Análisis Político en perspectiva comparada. Revista SAAP, Buenos Aires, v. 6, n. 2, p. 345-354, nov. 2012.

SARTORI, Giovanni. La política. Lógica y método en las ciencias sociales. México: Fondo de Cultura Económica, 1984.

¿Hacia dónde va la ciencia política? Política y gobierno, México, v. 11, n. 2, p. 349-354, segundo semestre, 2004.

SCHEDLER, Andreas. La relevancia pública de la política comparada. POSTData, Buenos Aires, n. 10, p. 341-349, dic. 2004.

SCHMITTER Philippe C. The Nature and Future of Comparative Politics. European Political Science Review, v. 1, n. 1, p. 31-61, 2009.

SHAPIRO, Ian. Problems, Methods, and Theories in The Study of Politics, or What's Wrong With Political Science and What to Do about it. Political Theory, v. 30, n. 4, p. 596-619, agosto, 2002.

STUMPF GONZÁLEZ, Rodrigo. A Agenda de Ensino e Pesquisa em Política Comparada: o caso Brasileiro. In: Jornada de debate sobre la Política Comparada en América Latina, Rosario, 2011.

TICKNER, Arlene B.; CEPEDA, Carolina; BERNAL, José Luis. Enseñanza. investigación y política internacional (TRIP) en América Latina. Documento de trabajo, Bogotá, n. 19, 2012.

TORRE, Juan Carlos. A la sombra de la revolución y la dictadura. Una reflexión sobre la ciencia política en Argentina como empresa intelectual. POSTData, Buenos Aires, n. 12, p. 207-212, ago. 2007.

URBANI, Giuliano. Política comparada. In: BOBBIO, Norberto; MATTEUCCI, Nicola; PASQUINO, Gianfranco. Diccionario de política, México: Siglo XXI, 1997. p. 1225-1230. 
